\title{
Generalized second-order directional derivatives and optimality conditions
}

\author{
$X . Q$. YANG
}

The purpose of this thesis is to study generalized second-order directional derivatives and generalized calculus for the class of $C^{1,1}$ functions, that is, continuously Gâteaux differentiable functions with locally Lipschitz gradients, to develop secondorder optimality conditions for optimization problems with $C^{1,1}$ functions and to explore optimization methods for solving such optimization problems.

A new generalized second-order directional derivative and a set-valued generalized Hessian are introduced for $C^{1,1}$ functions in Banach spaces. It is shown that this generalized Hessian is single-valued at a point if and only if the function is twice weakly Gâteaux differentiable. This generalized Hessian is contained in other recent generalized Hessians and so it provides tight second-order necessary conditions. Moreover, various connections between the generalized second-order directional derivatives and the parabolic second-order directional derivatives are established for $C^{1,1}$ functions. These connections enable us to compare the corresponding second-order optimality conditions. Chain rules for compositons of a $C^{1,1}$ function and a strengthened twice weakly Gâteaux differentiable function and of $C^{1,1}$ functions are derived. Generalized Taylor's expansions are obtained and applied to establish second-order characterizations of convex $C^{1,1}$ functions.

Applications include the development of second order optimality conditions for unconstrained, constrained and convex composite minimization problems and the study of optimization methods where $C^{1,1}$ functions are involved. Second-order necessary conditions are obtained using first-order and second-order constraint qualification. Various new second-order sufficient conditions of strict local minima are presented using generalized Taylor's expansions. Convex composite minimization problems are studied where the objective function is a composition of finite-valued or non-finite-valued convex function and a $C^{1,1}$ function.

A generalized smoothing approximation method using a two-parameter approximation is examined for studying a nonsmooth composite problem. Error analyses are

Received 29th August, 1994

Thesis submitted to the University of New South Wales, March 1994. Degree approved, July 1994. Supervisor: Dr V. Jeyakumar.

Copyright Clearance Centre, Inc. Serial-fee code: 0004-9729/95 SA2.00+0.00. 
established under some verifiable conditions. It is also shown how the point that satisfies second-order necessary conditions of a constrained $C^{1,1}$ minimization problem can be calculated using extended exterior point methods. Second-order necessary conditions for penalty functions are easily obtained using the established chain rules.

Department of Mathematics

The University of Western Australia

Nedlands WA 6009

Australia 